\title{
Suzaku Highlights of Supernova Remnants
}

\section{Satoru Katsuda*i}

Institute of Space and Astronautical Science (ISAS), Japan Aerospace Exploration Agency

(JAXA), 3-1-1 Yoshinodai, Chuo, Sagamihara, Kanagawa 252-5210, Japan

E-mail: katsuda@astro.isas.jaxa.jp

\section{Hiroshi Tsunemi}

Department of Earth and Space Science, Osaka University, 1-1 Machikaneyama-cho, Toyonaka, Osaka 560-0043, Japan

E-mail: tsunemidess.sci.osaka-u.ac.jp

\begin{abstract}
Suzaku was the Japanese 5th X-ray astronomy satellite operated from 2005 July 10 to $2015 \mathrm{Au}$ gust 26. Its key features are high-sensitivity wide-band X-ray spectroscopy available with both the X-ray imaging CCD cameras and the non-imaging collimated hard X-ray detector. A number of interesting scientific discoveries have been achieved in various fields. Among them, I will focus on results on supernova remnants. The topics in this paper include (1) revealing distributions of supernova ejecta, (2) establishing over-ionized plasmas by discoveries of radiative-recombination continua, (3) constraining progenitors of Type Ia SNRs from $\mathrm{Mn} / \mathrm{Cr}$ and $\mathrm{Ni} / \mathrm{Fe}$ line ratios, and (4) searching for X-ray counterparts from unidentified HESS sources. These results are of high scientific importance in physics of supernova explosions, non-equilibrium plasmas, and cosmic-ray acceleration.
\end{abstract}

XI Multifrequency Behaviour of High Energy Cosmic Sources Workshop,

25-30 May 2015

Palermo, Italy

* Speaker.

${ }^{\dagger}$ A footnote may follow. 


\section{Supernova Remnants \& The Suzaku Satellite}

Supernova remnants (SNRs) are the aftermaths of energetic supernova explosions that announces deaths of stars. There are roughly 400 SNRs in our own galaxy and nearby galaxies. Thanks to their proximity, they are of high scientific interest, providing us with insights into SN explosion mechanisms as well as SN nucleosynthesis. They are also astrophysical laboratories to study non-equilibrium plasmas. Moreover, there is growing evidence that their shock waves (both blastwaves and reverse shocks) accelerate most of cosmic rays in the galaxy. SNRs can be seen in many wavelengths ranging from radio to TeV gamma-rays. The SNR plasmas are usually so hot $\left(10^{6-7} \mathrm{~K}\right)$ that they emit X-rays most efficiently.

Suzaku was the Japanese 5th X-ray astronomy satellite launched in 2005 July 10 [23], and terminated the operation officially on 2015 August 26. It is characterized by the high-sensitivity wide-band X-ray spectroscopy performed by X-ray imaging CCD cameras (XIS) [19] and nonimaging collimated hard X-ray detector (HXD) [33]. Suzaku was also equipped with the X-Ray Spectrometer which is a non-dispersive, high-resolution X-ray spectrometer. Despite its initial success such as achievements of an energy resolution of $7 \mathrm{eV}$ onboard [17], on 2005 August 8, a thermal short between the helium and neon tanks resulted in the liquid helium coolant venting to space, leaving that system inoperable. Nonetheless, Suzaku successfully delivered a number of important results, thanks to the XIS and HXD. We will review results on supernova remnants (SNRs) that are mainly derived from the XIS.

\section{Revealing Ejecta Distributions}

Since SNRs are observed as extended objects, we can directly reveal distributions of SN ejecta, which is a strong observational merit that cannot be attained with extragalactic SNe. Combined with the high spectral resolution of Suzaku's XIS, which enables us to separate line emission from different elements, we can reveal ejecta distributions for individual elements. This capability is important to constrain recent SN explosion models that predict a variety of distributions of SN ejecta, including Ni-rich jets plus O-rich torus structures [20, 37], large-scale finger structures due to standing accretion-shock instabilities [35], and ejecta-neutron star recoils [48]. In addition, SNRs offer unique sites to study mixing of SN ejecta not only during SN explosions but also into the interstellar medium (ISM) at a very late phase. Therefore, X-ray observations of SNRs are important in understanding metal-enrichment processes in the Universe, too.

Suzaku revealed detailed ejecta structures for a number of SNRs. Among them, we here focus on the results of the Cygnus Loop - one of the nearest and brightest SNRs in the X-ray sky. Since the Loop is quite large (a radius of $\sim 1.4^{\circ}$ ), we conducted a large, $\sim 2 \mathrm{Msec}$, Suzaku and XMMNewton observations to cover the entire remnant with sufficient depths that allow for spatiallyresolved spectroscopy. The fields of view are shown in Fig. 1 left. The mosaiced Suzaku and XMMNewton image is shown in Fig. 1 center, for which red, green, and blue correspond to the energy ranges of $0.2-0.6 \mathrm{keV}, 0.6-1.2 \mathrm{keV}$, and $1.2-2.0 \mathrm{keV}$, respectively. This figure shows that the central region is harder than the outermost regions, which is consistent with previous GINGA, ROSAT, and ASCA observations $[9,3,27]$. This implies that relatively hot SN ejecta, being surrounded by a 

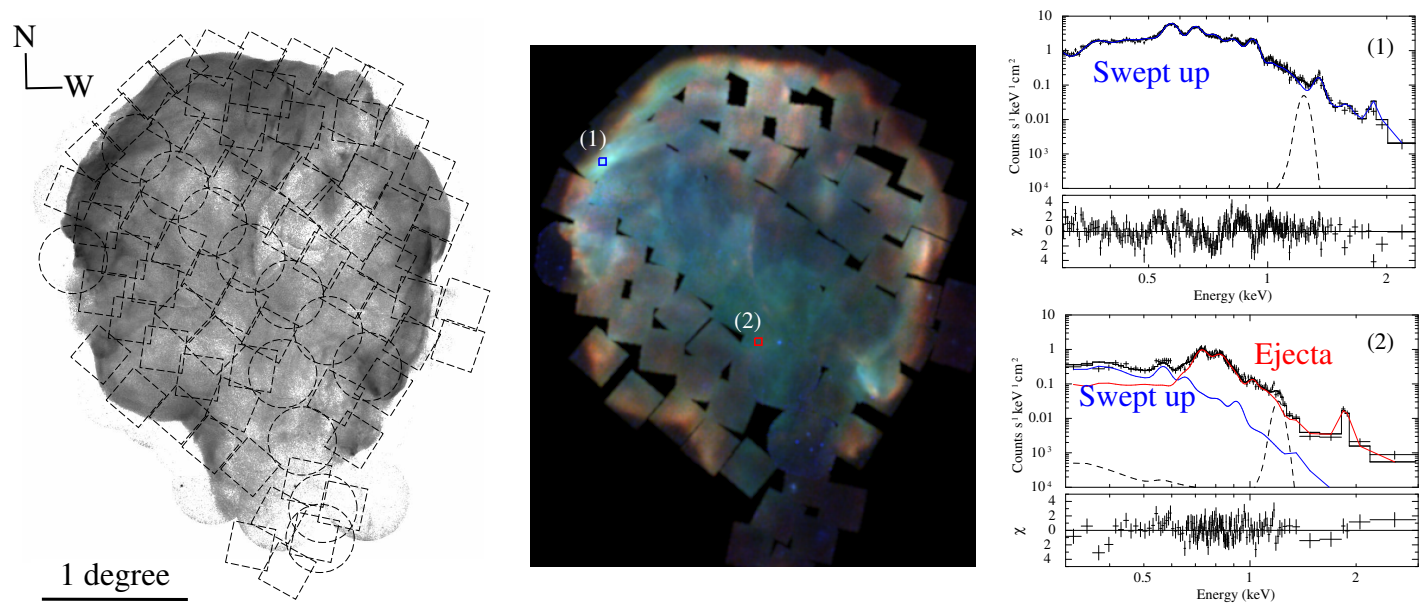

Figure 1: Left: X-ray image of the entire Cygnus Loop taken by ROSAT HRI. Overlaid boxes and circles are Suzaku's XIS and XMM-Newton's EPIC fields of view, respectively. Center: Combined XIS and EPIC image of the Cygnus Loop. Red, green, and blue correspond to the energy ranges of $0.2-0.6 \mathrm{keV}, 0.6-$ $1.2 \mathrm{keV}$, and $1.2-2.0 \mathrm{keV}$, respectively. The blue and red boxes, marked as (1) and (2), indicate regions where we extract spectra in the right panels. Right: XIS (BI) spectra from the regions (1) and (2) shown in the central panel. Blue and red represent swept-up and ejecta components, respectively. Lower panels show residuals.

relatively cool swept-up shell, occupy a large volume inside the remnant - a view constructed by spectroscopic studies with ASCA [25, 26].

To reveal detailed spatial structures of SN ejecta, we have performed spatially-resolved spectroscopy by dividing each field of view into small (a few arcminutes) rectangular regions. Two example XIS spectra taken from $3^{\prime} \times 3^{\prime}$ regions indicated in Fig. 1 center are presented in Fig. 1 right. The spectra can be fitted with either a single-component or two-components non-equilibrium ionization model, in which the low- and high-temperature components are supposed to be responsible for the swept-up medium and the SN ejecta, respectively. These models give reasonably good fits for all of the spectra, often requiring two-component model for the interior of the remnant [40, 12, 18, 41, 42]. As described in these papers, the distribution of ejecta is never uniform, but highly asymmetric in the sense that the ejecta are preferentially distributed in the southern part of the remnant.

All of the published results used incomplete data sets that were available at the analysis phases. We here use all the data sets in Fig. 1, and generate band ratio maps of O VII (0.5-0.6 keV), Mg XI (1.26-1.4 keV), Si XIII (1.7-2.0 keV) to a continuum (1.4-1.7 keV), as shown in Fig. 2. We can find clearly distinct distribution patterns; $\mathrm{O}$ is rim-brightened, whereas $\mathrm{Si}$ is concentrated into the central part, and $\mathrm{Mg}$ is comparatively uniform. This confirms that $\mathrm{O}$ is predominantly arising from the swept-up medium, while Si is dominated by $\mathrm{SN}$ ejecta, and $\mathrm{Mg}$ is intermediate. It should be also noted that we confirm clear asymmetric distributions of $\mathrm{Si}$, which are biased to the south and are extending to the southern blow-out region. We suggest that this asymmetry is 

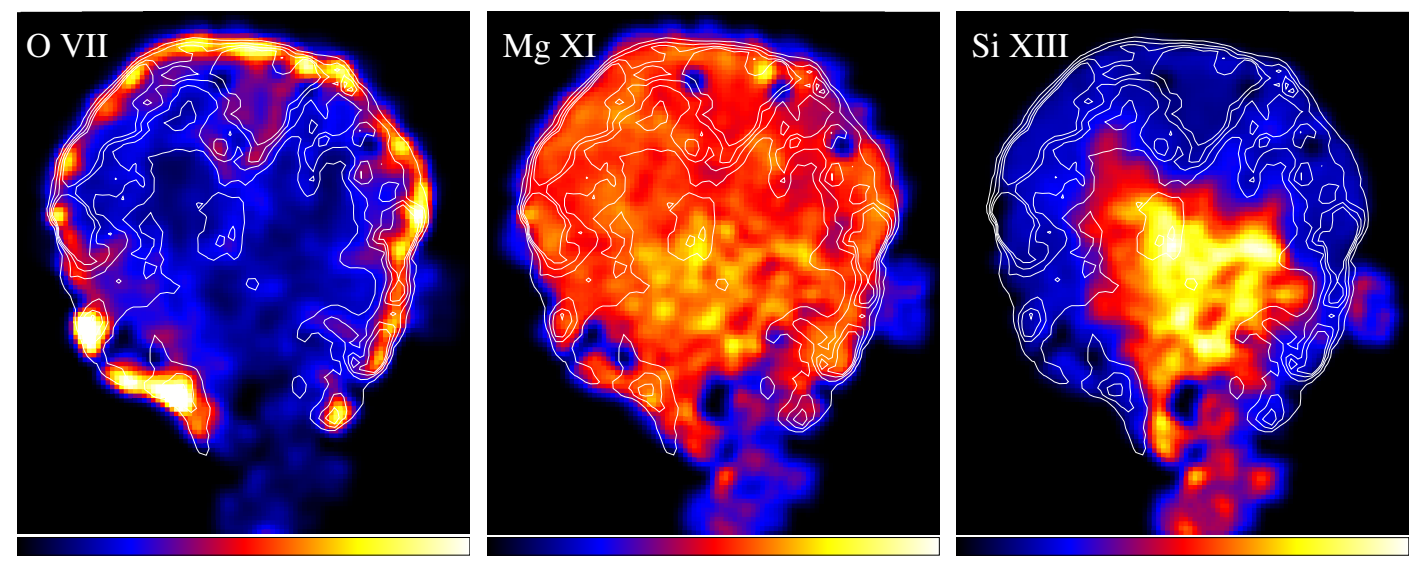

Figure 2: Linearly-scaled band-ratio maps of O VII K (0.5-0.6 keV), Mg XI K (1.26-1.4 keV), and Si XIII $\mathrm{K}(1.7-2.0 \mathrm{keV})$ to a continuum (1.4-1.7 keV) based on merged XIS and EPIC data. White contours overlaid represent X-ray intensities based on ROSAT all-sky survey data.

caused by intrinsic SN explosions rather than environmental effects; if the environmental effects were important, we would see oppositely biased ejecta distribution (to the north), since the reverse shock should proceed more in the north than in the south, given the presence of a dense wall in the north but a blow-out in the south. Subsequently, this kind of ejecta asymmetry has been revealed in many SNRs (both CC SNRs and Ia SNRs) including Puppis A [11, 14], G156.2+5.7 [13, 43], and SN 1006 [45], indicating the commonality of asymmetric, uni-polar SN explosions.

\section{Establishing Over-Ionized Plasmas}

The SNR plasmas are usually in non-equilibrium ionization states, unlike clusters of galaxies where we usually see relaxed plasmas. This is simply because the time after the shock heating in SNRs is much shorter than the equilibration timescale. In a SNR (collisionless) shock for which each elemental species is expected to attain the mass-proportional temperature, we expect the electron temperature to be much lower than the ion temperature right behind the shock. Then, the electron temperature gradually increases through interactions with ions. The hot electrons start to ionize the nuclei. Therefore, the ionization (rather than recombination) proceeds in SNRs, so that we should observe electron temperatures higher than what are expected from the ionization states. In fact, such under-ionized non-equilibrium conditions have been recognized in many SNRs since 1980's [47, 39, 24].

On the other hand, an opposite non-equilibration states, the so-called "over-ionized" state, in which a plasma has a higher ionization state than what is expected from an electron temperature, was claimed in IC 443 and W49B, based on anomalously high K-shell lines in H-like ions compared with those in He-like ions $[15,16]$. However, later XMM-Newton studies were able to explain the X-ray spectrum with an equilibrum emission model, questioning the "over-ionized" states [38].

Suzaku provided new insights into this problem, based on the discovery of strong radiativerecombination continua (RRC) from IC 443 [49]. The line ratios as well as the RRC features 
are self-consistently explained by a recombining (or over-ionized) plasma. After this discovery, roughly ten SNRs were found to show recombining plasmas evidenced by the RRC features [29, $28,32,44,51]$. It should be noted that the over-ionized plasmas have been found only from mixedmorphology SNRs, i.e., SNRs assoiciated with radio shells plus centrally-peaked thermal X-rays [31]. This point might be the key to understand when and how these over-ionized plasmas were formed.

\section{Probing Type Ia Progenitors by Line Emission from Cr, Mn, Fe, and Ni}

Suzaku opened a new window to detect faint line emission from minor iron-group elements such as $\mathrm{Cr}, \mathrm{Mn}$, and $\mathrm{Ni}$, whose intensity ratios are sensitive to the properties of $\mathrm{SNe}$ Ia progenitors. Badenes et al.(2008) [4] noticed that the $\mathrm{Mn} / \mathrm{Cr}$ ratio strongly depends on the metallicity of an exploding white dwarf. Briefly, neutron excess of $\mathrm{Mn}$ (with respect to $\mathrm{Cr}$ ) is an important clue to understanding the physics. Since the composition of a white dwarf is $\mathrm{C}, \mathrm{O}$, and a little bit neutronrich ${ }^{22} \mathrm{Ne}$, more ${ }^{22} \mathrm{Ne}$ would produce more $\mathrm{Mn}$. Meanwhile, ${ }^{22} \mathrm{Ne}$ originates from $\mathrm{N}$, which is the product of CNO-cycle during H-burning in a main sequence star. Since $\mathrm{CNO}$-cycle converts $\mathrm{C}$ and $\mathrm{O}$ into $\mathrm{N}$ (practically), the amount of $\mathrm{C}$ and $\mathrm{O}$ controls the amount of $\mathrm{N}$ as well as neutron-excess elements like ${ }^{22} \mathrm{Ne}, \mathrm{Mn}$, and $\mathrm{Ni}$. If we assume that the amount of $\mathrm{C}$ and $\mathrm{O}$ represents the metallicity, then we should expect a positive correlation between the progenitor meteallicity and $\mathrm{Mn} / \mathrm{Cr}$ and $\mathrm{Ni} / \mathrm{Fe}$ ratios in the SN ejecta. Based on this method, the progenitor metallicities of Tycho's SNR and Kepler's SNR were estimated to be $\sim 1$ and $\sim 3$ times the solar values, respectively [36, 30].

Recently, an extremely high $\mathrm{Ni} / \mathrm{Fe}$ and $\mathrm{Mn} / \mathrm{Fe}$ line ratios (or mass ratios of $0.11-0.24$ and $0.018-0.033$, respectively) were found in $3 \mathrm{C} 397$ [50]. The high ratios are hard to be reconciled solely by controlling the metallicity of the progenitor star. Therefore, Yamaguchi et al. (2015) postulated that electron-caputure processes inside the exploding white dwarf make the extreme neutron excess. For the electron-caputure process to occur, the core density of the white dwarf needs to be higher than $2 \times 10^{8} \mathrm{~g} \mathrm{~cm}^{-3}$ which can be attained only if white dwarfs are heavier than $1.2 \mathrm{M}_{\odot}$. Thus, in this scenario, the classical single-genenrate channel, in which a Chandrasekharmass white dwarf is involved, is preferred as a progenitor star.

\section{Identifying Unidentified TeV Gamma-Ray Sources}

The H.E.S.S. collaboration has performed a survey of the Galactic plane, discovering tens of new sources in $\mathrm{TeV}$ gamma-rays [1]. Interestingly, about 50 sources were found not to be associated with known sources in other wavelengths [10]. They stay unidentified, and are called "TeV unID sources" or "dark particle accelerators". If they really do not have counterparts in Xrays (synchrotron radiation), they are most likely to be proton accelerators, i.e., origins of cosmic rays. Therefore, deep follow-up observations are eargely awaited.

To date, X-ray follow-ups have been performed for roughly half of these unID sources. As a result, many of them were identified as pulsar wind nebulae (PWNe). These include HESS J1614518 [22], HESS J1825-137 [46], HESS J1837-069 [2], and several other sources identified with XMM-Newton and Chandra. Since PWNe do not likely accelerate protons, they can not be origins of cosmic rays. 
On the other hand, Suzaku gave strict upper limits for some other sources, including HESS J1616508 [21], HESS J1745-303 [6], and HESS J1804-216 [5]. Interestingly, Fermi detected strong GeV gamma-ray emission from HESS J1616-508 and HESS J1804-216, which can be interpreted as the pion-decay bump due to interactions between accelerated nucleons with the ambient matter [7]. These sources are therefore promising candidates of proton accelerators. However, their photon indices would be too steep to produce observed cosmic-ray spectrum, if we consider a possible spectral softening during cosmic-ray propagation to the earth. Therefore, further observations are desired to understand their nature(s). In particular, it is essential to fill the current observation gap between $10 \mathrm{keV}$ and $1 \mathrm{GeV}$, part of which will be covered with NuSTAR [8] and ASTRO-H [34].

\section{References}

[1] Aharonian, F., Akhperjanian, A.G., Aye, K.-M., et al. 2005 A New Population of Very High Energy Gamma-Ray Sources in the Milky Way, Science, 307, 1938

[2] Anada, T., Ebisawa, K., Dotani, T., \& Bamba, A. 2009, X-Ray Studies of HESS J1837-069 with Suzaku and ASCA: a VHE Gamma-Ray Source Originated from the Pulsar Wind Nebula PASJ, 61, S183

[3] Aschenbach, B., \& Leahy, D.A. 1999, ROSAT all-sky survey map of the Cygnus Loop: Overall structure and comparison with radio map, A\&A, 341, 602

[4] Badenes, C., Bravo, E., \& Hughes, J.P. 2008, The End of Amnesia: A New Method for Measuring the Metallicity of Type Ia Supernova Progenitors Using Manganese Lines in Supernova Remnants, ApJL, 680, L33

[5] Bamba, A., Koyama, K., Hiraga, J.S. 2007, Discovery of a Possible X-Ray Counterpart to HESS J1804-216, PASJ, 59, S209

[6] Bamba, A., Yamazaki, R., Kohri, K., et al. 2009, X-ray Observation of Very High Energy Gamma-Ray Source, HESS J1745-303, with Suzaku, ApJ, 691, 1854

[7] Bamba, A. 2010, High energy aspects of SNRs, AIP Conference Proceeding, 1248, 33

[8] Harrison, F.A., Craig, W.W., Christensen, F.E. 2013, The Nuclear Spectroscopic Telescope Array (NuSTAR) High-energy X-Ray Mission, ApJ, 770, 103

[9] Hatsukade, I., \& Tsunemi, H. 1990, Spatial structure of the Cygnus loop in the X-ray region above 1.5 keV, PASJ, 362, 566

[10] Hinton, J.A., \& Hofmann, W. 2009, Teraelectronvolt Astronomy, ARA\&A, 47, 523

[11] Hwang, U., Petre, R., Flanagan, K.A. 2008, X-Ray-emitting Ejecta in Puppis A Observed with Suzaku, ApJ, 676, 378

[12] Katsuda, S., Tsunemi, H., Miyata, E., et al. 2008, Asymmetric Ejecta Distribution of the Cygnus Loop Revealed with Suzaku, PASJ, 60, S107

[13] Katsuda, S., Petre, R., Hwang, U., et al. 2009, Suzaku Observations of Thermal and Non-Thermal X-Ray Emission from the Middle-Aged Supernova Remnant G156.2+5.7, PASJ, 61, S155

[14] Katsuda, S., Hwang, U., Petre, R., et al. 2010, Discovery of X-ray-emitting O-Ne-Mg-rich Ejecta in the Galactic Supernova Remnant Puppis, ApJ, 714, 1725 
[15] Kawasaki, M. Ozaki, M., Nagase, F., et al. 2002 ASCA Observations of the Supernova Remnant IC 443: Thermal Structure and Detection of Overionized Plasma, ApJ, 572, 897

[16] Kawasaki, M. Ozaki, M., Nagase, F., Inoue, H., \& Petre, R. 2005, Ionization States and Plasma Structures of Mixed-Morphology Supernova Remnants Observed with ASCA, ApJ, 631, 935

[17] Kelley, R.L., Mitsuda, K., Allen, C.A., et al. 2007, The Suzaku High Resolution X-Ray Spectrometer, PASJ, 59, S77

[18] Kimura, M., Tsunemi, H., Katsuda, S., Uchida, H. 2009, Suzaku Observations across the Cygnus Loop from the Northeastern to the Southwestern Rim, PASJ, 61, S137

[19] Koyama, K., Tsunemi, H., Dotani, T., et al. 2007, X-Ray Imaging Spectrometer (XIS) on Board Suzaku, PASJ, 59, S23

[20] Maeda, Keiichi, \& Nomoto, Ken'ichi 2003, Bipolar Supernova Explosions: Nucleosynthesis and Implications for Abundances in Extremely Metal-Poor Stars, ApJ, 598, 1163

[21] Matsumoto, H., Ueno, M., Bamba, A., et al. 2007 Suzaku Observations of HESS J1616-508: Evidence for a Dark Particle Accelerator, PASJ, 59, S199

[22] Matsumoto, H., Uchiyama, H., Sawada, M., et al. 2008, Suzaku Observations of HESS J1616-508: Evidence for a Dark Particle Accelerator, PASJ, 60, S163

[23] Mitsuda K., Bautz M., Inoue H., et al. 2007, The X-Ray Observatory Suzaku, PASJ, 59, S1

[24] Miyata, E., Tsunemi, H., Pisarski, R., \& Kissel, S.E. 1994, The plasma structure of the north-east rim of the Cygnus Loop as observed with ASCA, PASJ, 46, L101

[25] Miyata, E., Tsunemi, H., Kohmura, T., Suzuki, S., \& Kumagai, S. 1998, Metal-Rich Plasma at the Center Portion of the Cygnus Loop, PASJ, 50, 257

[26] Miyata, E., \& Tsunemi, H. 1999, The Radial Structure of the Cygnus Loop Supernova Remnant: Possible Evidence of a Cavity Explosion, ApJ, 525, 305

[27] Miyata, E., Tsunemi, H., Koyama, K., \& Ishisaki, Y. 2000, ASCA observation of the Cygnus Loop Supernova Remnant, Advances in Space Research, 25, 558

[28] Ohnishi, T., Koyama, K., Tsuru, T., et al. 2011, X-Ray Spectrum of a Peculiar Supernova Remnant, G359.1-0.5, PASJ, 63, 527

[29] Ozawa, M., Koyama, K., Yamaguchi, H., Masai, K., \& Tamagawa, T. 2009, Suzaku Discovery of the Strong Radiative Recombination Continuum of Iron from the Supernova Remnant W49B, ApJ, 706, L71

[30] Park, S., Badenes, C., Mori, K., et al. 2013, A Super-solar Metallicity for the Progenitor of Kepler's Supernova, ApJL, 767, L10

[31] Rho, J., \& Petre, R. 1998, Mixed-Morphology Supernova Remnants, ApJL, 503, L167

[32] Sawada, M., \& Koyama, K. 2012, X-Ray Observations of the Supernova Remnant W28 with Suzaku. I. Spectral Study of the Recombining Plasma, PASJ, 64, 81

[33] Takahashi, T., Abe, K., Endo, M., et al. 2007, Hard X-Ray Detector (HXD) on Board Suzaku, PASJ, 59, S35

[34] Takahashi, T., Mitsuda, K., Kelley, R., et al. 2014 The ASTRO-H X-ray astronomy satellite Proceedings of the SPIE, 9144, 914425 
[35] Takiwaki, T., Kotake, K., Suwa, Y. 2012, Three-dimensional Hydrodynamic Core-collapse Supernova Simulations for an 11.2 $M_{\odot}$ Star with Spectral Neutrino, ApJ, 749, 98

[36] Tamagawa, T., Hayato, A., Nakamura, S., et al. 2009, Suzaku Observations of Tycho's Supernova Remnant, PASJ, 61, S167

[37] Tominaga, N., Maeda, K., Umeda, H., et al. 2003, The Connection between Gamma-Ray Bursts and Extremely Metal-poor Stars: Black Hole-forming Supernovae with Relativistic Jets, ApJL, 657, L77

[38] Troja, E., Bocchino, F., Miceli, M., \& Reale, F. 2008, XMM-Newton observations of the supernova remnant IC 443. II. Evidence of stellar ejecta in the inner regions, A\&A, 485, 777

[39] Tsunemi, H., Yamashita, K., Masai, K., Hayakawa, S., \& Koyama, K. 1986, X-ray spectra of the Cassiopeia A and TYCHO supernova remnants and their element abundances, ApJ, 306, 248

[40] Tsunemi, H., Katsuda, S., Nemes, N., \& Miller, E.D. 2007, The Plasma Structure of the Cygnus Loop from the Northeastern Rim to the Southwestern Rim, ApJ, 671, 1717

[41] Uchida, H., Tsunemi, H., Katsuda, S., et al. 2009, Ejecta Distributions of Heavy Elements in the Cygnus Loop, PASJ, 61, 301

[42] Uchida, H., Tsunemi, H., Katsuda, S., et al. 2009, Line-of-Sight Shell Structure of the Cygnus Loop, PASJ, 61, 503

[43] Uchida, H., Tsunemi, H., Katsuda, S., et al. 2012, Recombining Plasma and Hard X-Ray Filament in the Mixed-Morphology Supernova Remnant W 44, PASJ, 64, 61

[44] Uchida, H., Koyama, K., Yamaguchi, H., et al. 2012, Recombining Plasma and Hard X-Ray Filament in the Mixed-Morphology Supernova Remnant W 44, PASJ, 64, 141

[45] Uchida, H., Yamaguchi, H., Koyama, K. 2013, Asymmetric Ejecta Distribution in SN 1006, ApJ, 771, 56

[46] Uchiyama, H., Matsumoto, H., Tsuru, T.G., Koyama, K., \& Bamba, A. 2009, Suzaku Observation of HESS J1825-137: Discovery of Largely-Extended X-Rays from PSR J1826-1334 PASJ, 61, S189

[47] Winkler, P.F., Clark, G.W., Markert, T.H., Petre, R., \& Canizares, C.R. 1981, X-ray line emission from the Puppis A supernova remnant - Oxygen lines, ApJ, 245, 574

[48] Wongwathanarat, A., Janka, H.-Th., Muller, E. 2013, Three-dimensional neutrino-driven supernovae: Neutron star kicks, spins, and asymmetric ejection of nucleosynthesis products, A\&A, 552, 126

[49] Yamaguchi, H., Ozawa, M., Koyama, K., et al. 2009, Discovery of Strong Radiative Recombination Continua from the Supernova Remnant IC 443 with Suzaku, ApJ, 705, L6

[50] Yamaguchi, H., Badenes, C., Foster, A., et al. 2015, A Chandrasekhar Mass Progenitor for the Type Ia Supernova Remnant 3C 397 from the Enhanced Abundances of Nickel and Manganese, ApJ, 801, L31

[51] Yamauchi, S., Nobukawa, M., Koyama, K., \& Yonemori, M. 2013, Evidence for Recombining Plasma in the Supernova Remnant G346.6-0.2, PASJ, 65, 6 